PROCEEDINGS OF THE

AMERICAN MATHEMATICAL SOCIETY

Volume 135, Number 2, February 2007, Pages 531-540

S 0002-9939(06)08487-5

Article electronically published on August 2, 2006

\title{
REGULARITY OF SOLUTIONS TO STOCHASTIC VOLTERRA EQUATIONS WITH INFINITE DELAY
}

\author{
ANNA KARCZEWSKA AND CARLOS LIZAMA
}

(Communicated by Richard C. Bradley)

\begin{abstract}
In this article we give necessary and sufficient conditions providing regularity of solutions to stochastic Volterra equations with infinite delay on a $d$-dimensional torus. The harmonic analysis techniques and stochastic integration in function spaces are used. The work applies to both the stochastic heat and wave equations.
\end{abstract}

\section{INTRODUCTION}

We consider the following integro-differential stochastic equation with infinite delay:

$$
X(t, \theta)=\int_{-\infty}^{t} b(t-s)\left[\Delta X(s, \theta)+\frac{\partial W_{\Gamma}}{\partial s}(s, \theta)\right] d s, \quad t \geq 0, \quad \theta \in T^{d},
$$

where $b \in L^{1}\left(\mathbb{R}_{+}\right), \Delta$ is the Laplace operator and $T^{d}$ is the $d$-dimensional torus. In (1.1), $W_{\Gamma}$ is a spatially homogeneous Wiener process with the space covariance $\Gamma$ and taking values in the space of tempered distributions $\mathcal{S}^{\prime}\left(T^{d}\right)$. Such an equation arises, in the deterministic case, in the study of heat flow in materials of fading memory type (see [3], [14]).

In this paper we address the following question: under what conditions on the covariance $\Gamma$ does the process $X$ take values in a Sobolev space $H^{\alpha}\left(T^{d}\right)$, particularly in $L^{2}\left(T^{d}\right)$ ?

This problem has been investigated, for the stochastic heat and wave equations in case of $\mathbb{R}^{2}$, by Dalang and Frangos [4] (see also Mueller [13]). Their results were recently extended on $\mathbb{R}^{d}$ and generalized to $T^{d}$ in [9]. In [10, regularity of stochastic convolutions solving Volterra equations on $\mathbb{R}^{d}$ has been studied. The results have been obtained by using the resolvent operators corresponding to Volterra equations and expressed in terms of the spectral measure and the covariance kernel $\Gamma$ of the Wiener process $W_{\Gamma}$. Our approach is different. We study a particular case of weak solutions under the basis of an explicit representation of the solution to (1.1) (cf. formula (3.6)). We remark that the knowledge of the regularity of solutions is important in the study of nonlinear stochastic equations (see, e.g., [4] and [12]).

Received by the editors April 15, 2005 and, in revised form, August 25, 2005.

2000 Mathematics Subject Classification. Primary 60H20; Secondary 60H05, 45D05.

Key words and phrases. Stochastic Volterra equation, function-valued solutions, equations on a torus, spatially homogeneous Wiener process.

The second author was supported in part by FONDECYT Grant \#1050084.

(C)2006 American Mathematical Society Reverts to public domain 28 years from publication 
Observe that (1.1) can be viewed as the limiting equation for the stochastic Volterra equation

$$
X(t, \theta)=\int_{0}^{t} b(t-s)\left[\Delta X(s, \theta)+\frac{\partial W_{\Gamma}}{\partial s}(s, \theta)\right] d s, \quad t \geq 0, \quad \theta \in T^{d} ;
$$

see [15, Chapter III, Section 11.5] to obtain details on this assertion. If $b$ is sufficiently regular, we get, by differentiating (1.2) with respect to $t$,

$$
\frac{\partial X}{\partial t}(t, \theta)=b(0)\left[\Delta X(t, \theta)+\frac{\partial W_{\Gamma}}{\partial t}(t, \theta)\right]+\int_{0}^{t} b^{\prime}(t-s)\left[\Delta X(s, \theta)+\frac{\partial W_{\Gamma}}{\partial s}(s, \theta)\right] d s
$$

where $t \geq 0$ and $\theta \in T^{d}$.

Taking in (1.3) $b(t) \equiv 1$, we obtain

$$
\left\{\begin{aligned}
\frac{\partial X}{\partial t}(t, \theta) & =\Delta X(t, \theta)+\frac{\partial W_{\Gamma}}{\partial t}(t, \theta), \quad t>0, \theta \in T^{d} \\
X(0, \theta) & =0, \quad \theta \in T^{d} .
\end{aligned}\right.
$$

Similarly, taking $b(t) \equiv t$ and differentiating (1.2) twice with respect to $t$ we obtain

$$
\left\{\begin{aligned}
\frac{\partial^{2} X}{\partial t^{2}}(t, \theta) & =\Delta X(t, \theta)+\frac{\partial W_{\Gamma}}{\partial t}(t, \theta), \quad t>0, \theta \in T^{d}, \\
X(0, \theta) & =0, \quad \theta \in T^{d}, \\
\frac{\partial X}{\partial t}(0, \theta) & =0, \quad \theta \in T^{d} .
\end{aligned}\right.
$$

It has been shown in [9, Theorem 5.1] (see also [8, Theorem 1]) that (1.4) and (1.5) on the $d$-dimensional torus $T^{d}$ have an $H^{\alpha+1}\left(T^{d}\right)$-valued solution if and only if the Fourier coefficients $\left(\gamma_{n}\right)$ of the space covariance $\Gamma$ of the process $W_{\Gamma}$ satisfy

$$
\sum_{n \in \mathbb{Z}^{d}} \gamma_{n}\left(1+|n|^{2}\right)^{\alpha}<\infty
$$

Observe that for both stochastic heat (1.4) and wave (1.5) equations, the conditions are exactly the same, despite the different nature of the equations. On the other hand, the obtained characterization gives a natural framework in which nonlinear heat and wave equations can be studied.

In this article, we will prove that condition (1.6) even characterizes $H^{\alpha+1}\left(T^{d}\right)$ valued solutions for the stochastic Volterra equation (1.1), provided certain conditions on the kernel $b$ are satisfied. This is a strong contrast with the deterministic case, where regularity of (1.1) is dependent on the kernel $b$. The conditions that we impose on $b$ are satisfied by a large class of functions. Moreover, the important examples $b(t)=e^{-t}$ and $b(t)=t e^{-t}$ are shown to satisfy our assumptions.

We use, instead of resolvent families, a direct approach to the equation (1.1) finding an explicit expression for the solution in terms of the kernel $b$. This approach reduces the considered problem to questions in harmonic analysis and leads us to a complete answer.

\section{Preliminaries}

Let $\left(\Omega, \mathcal{F},\left(\mathcal{F}_{t}\right)_{t \geq 0}, P\right)$ be a complete filtered probability space. By $T^{d}$ we denote the $d$-dimensional torus which can be identified with the product $(-\pi, \pi]^{d}$. Let $D\left(T^{d}\right)$ and $D^{\prime}\left(T^{d}\right)$ denote, respectively, the topological vector space of functions $\phi \in C_{0}^{\infty}\left(T^{d}\right)$ endowed with the usual topology corresponding to the convergence (see, [1] or [17) and the space of distributions. By $\langle\xi, \phi\rangle$ we denote the value of a distribution $\xi$ on a test function. We assume that $W_{\Gamma}$ is a $D^{\prime}\left(T^{d}\right)$-valued spatially 
homogeneous Wiener process (abbr. s.h. Wiener process) with covariance $\Gamma$. For more information about the s.h. Wiener process we refer to [10].

Any arbitrary s.h. Wiener process $W_{\Gamma}$ is uniquely determined by its covariance $\Gamma \in D^{\prime}\left(T^{d}\right)$, which is a positive definite distribution, according to the formula

$$
\mathbb{E}\left\langle W_{\Gamma}(t, \theta), \phi\right\rangle\left\langle W_{\Gamma}(\tau, \theta), \psi\right\rangle=\min (t, \tau)\left\langle\Gamma, \phi \star \psi_{(s)}\right\rangle,
$$

where $\phi, \psi \in D\left(T^{d}\right)$ and $\psi_{(s)}(\eta)=\psi(-\eta)$, for $\eta \in T^{d}$. Because $W_{\Gamma}$ is a spatially homogeneous process, the distribution $\Gamma=\Gamma(\theta-\eta)$ for $\theta, \eta \in T^{d}$.

The space covariance $\Gamma$, like distribution in $D^{\prime}\left(T^{d}\right)$, may be uniquely expanded (see, e.g., [5 or 17]) into its Fourier series (with parameter $w=1$ because the period is $2 \pi$ )

$$
\Gamma(\theta)=\sum_{n \in \mathbb{Z}^{d}} e^{i(n, \theta)} \gamma_{n}, \quad \theta \in T^{d},
$$

convergent in $D^{\prime}\left(T^{d}\right)$. In (2.2),$(n, \theta)=\sum_{i=1}^{d} n_{i} \theta_{i}$ and $\mathbb{Z}^{d}$ denotes the product of integers.

The coefficients $\gamma_{n}$, in the Fourier series (2.2), satisfy (see, e.g., [5, Lesson 29, Section 29.4] or [9]):

(1) $\gamma_{n}=\gamma_{-n}$ for $n \in \mathbb{Z}^{d}, \gamma_{n}$ are non-negative,

(2) the sequence $\left(\gamma_{n}\right)$ is slowly increasing, that is,

$$
\sum_{n \in \mathbb{Z}^{d}} \frac{\gamma_{n}}{1+|n|^{r}}<+\infty, \text { for some } r>0 .
$$

Let us introduce, by induction, the following set of indexes. Denote $\mathbb{Z}_{s}^{1}:=\mathbb{N}$, the set of natural numbers, and define $\mathbb{Z}_{s}^{d+1}:=\left(\mathbb{Z}_{s}^{1} \times \mathbb{Z}^{d}\right) \cup\left\{(0, n): n \in \mathbb{Z}_{s}^{d}\right\}$. Let us note that $\mathbb{Z}^{d}=\mathbb{Z}_{s}^{d} \cup\left(-\mathbb{Z}_{s}^{d}\right) \cup\{0\}$. For instance, for $d=2, \mathbb{Z}_{s}^{2}=\mathbb{N} \times \mathbb{Z} \cup\{(0, n): n \in \mathbb{Z}\}$.

Now, the s.h. Wiener process $W_{\Gamma}$ corresponding to the covariance $\Gamma$ given by (2.2) may be represented in the form

$$
\begin{array}{r}
W_{\Gamma}(t, \theta)=\sqrt{\gamma_{0}} \beta_{0}(t)+\sum_{n \in \mathbb{Z}_{s}^{d}} \sqrt{2 \gamma_{n}}\left[\cos (n, \theta) \beta_{n}^{1}(t)+\right. \\
\left.+\sin (n, \theta) \beta_{n}^{2}(t)\right], \\
t \geq 0 \text { and } \theta \in T^{d} .
\end{array}
$$

In (2.4) $\beta_{0}, \beta_{n}^{1}, \beta_{n}^{2}, n \in \mathbb{Z}_{s}^{d}$, are independent real Brownian motions and $\gamma_{0}, \gamma_{n}$ are coefficients of the series (2.2). The series (2.4) is convergent in the sense of $D^{\prime}\left(T^{d}\right)$.

Because any periodic distribution with positive period is a tempered distribution (see, e.g., [5]), we may restrict our considerations to the space $S^{\prime}\left(T^{d}\right)$ of tempered distributions. By $S\left(T^{d}\right)$ we denote the space of infinitely differentiable rapidly decreasing functions on the torus $T^{d}$.

In this paper we use the following definition of the Sobolev spaces. By $H^{\alpha}=$ $H^{\alpha}\left(T^{d}\right), \alpha \in \mathbb{R}$, we denote the real Sobolev space of order $\alpha$ on the torus $T^{d}$. The norms in such spaces may be expressed in terms of the Fourier coefficients (see [1])

$$
\|\xi\|_{H^{\alpha}}=\left(\sum_{n \in \mathbb{Z}^{d}}\left(1+|n|^{2}\right)^{\alpha}\left|\xi_{n}\right|^{2}\right)^{\frac{1}{2}}=\left(\left|\xi_{0}\right|^{2}+2 \sum_{n \in \mathbb{Z}_{s}^{d}}\left(1+|n|^{2}\right)^{\alpha}\left(\left(\xi_{n}^{1}\right)^{2}+\left(\xi_{n}^{2}\right)^{2}\right)\right)^{\frac{1}{2}},
$$

where $\xi_{n}=\xi_{n}^{1}+i \xi_{n}^{2}, \xi_{=} \bar{\xi}_{-n}, n \in \mathbb{Z}^{d}$.

There is another possibility to define the Sobolev spaces (see, e.g., [16]). 


\section{Main Results}

If $b \in L_{\text {loc }}^{1}\left(\mathbb{R}_{+}\right)$and $\mu \in \mathbb{C}$, we shall denote by $r(t, \mu)$ the unique solution in $L_{\text {loc }}^{1}\left(\mathbb{R}_{+}\right)$to the linear Volterra equation

$$
r(t, \mu)=b(t)+\mu \int_{0}^{t} b(t-s) r(s, \mu) d s, \quad t \geq 0 ;
$$

see [7, Theorem 3.1]. By taking the Laplace transform in $t$ we obtain $r(\lambda, \mu)=$ $\hat{b}(\lambda)(1-\mu \hat{b}(\lambda))^{-1}$ and hence, in many cases, the function $r(t, \mu)$ may be found explicitly. For example, if $b(t) \equiv 1$, then $r(\lambda, \mu)=\frac{1}{\lambda-\mu}$ or, inverting the Laplace transform, we obtain $r(t, \mu)=e^{\mu t}$. Analogously, if $b(t)=e^{-t}$, then $r(\lambda, \mu)=$ $\frac{1}{\lambda+(1-\mu)}$ and hence $r(t, \mu)=e^{(-1+\mu) t}$. Other examples are:

$$
\begin{array}{ll}
b(t)=t, & r(t, \mu)=\frac{\sinh \sqrt{\mu} t}{\sqrt{\mu}} \quad \mu \neq 0, \\
b(t)=t e^{-t}, & r(t, \mu)=e^{-t} \frac{\sinh \sqrt{\mu} t}{\sqrt{\mu}}, \quad \mu \neq 0 .
\end{array}
$$

For more examples, see monograph 15 by Prüss.

Let us denote by $\hat{f}(k), k \in \mathbb{Z}$, the $k$-th Fourier coefficient of an integrable function $f$ :

$$
\hat{f}(k)=\frac{1}{2 \pi} \int_{0}^{2 \pi} e^{-i k t} f(t) d t .
$$

Given $f$ defined on $[0,2 \pi]$ we again denote by $f$ the periodic extension to $\mathbb{R}$. Let $b \in L^{1}\left(\mathbb{R}_{+}\right)$. We first observe from

$$
F(t):=\int_{-\infty}^{t} b(t-s) f(s) d s=\int_{0}^{\infty} b(s) f(t-s) d s
$$

that $F$ is periodic of period $T=2 \pi$ as is $f$. Now using Fubini's theorem we obtain,

$$
\hat{F}(k)=\tilde{b}(i k) \hat{f}(k), \quad k \in \mathbb{Z},
$$

where $\tilde{b}(\lambda):=\int_{0}^{\infty} e^{-\lambda t} b(t) d t$ denotes the Laplace transform of $b$.

In what follows we will assume that $\tilde{b}(i k)$ exists for all $k \in \mathbb{Z}$ and suppose that $\lambda \rightarrow \tilde{b}(\lambda)$ admits an analytical extension to a sector containing the imaginary axis, and still denote this extension by $\tilde{b}$. We introduce the following definition.

Definition 3.1. Let $r$ be given as in (3.1). We say that a kernel $b \in L^{1}\left(\mathbb{R}_{+}\right)$is admissible for (1.1) if

$$
\lim _{|n| \rightarrow \infty}|n|^{2} \int_{0}^{\infty}\left[r\left(s,-|n|^{2}\right)\right]^{2} d s=: C_{b}
$$

exists.

Example 3.2. i) In case $b(t)=e^{-t}$ we obtain

$$
|n|^{2} \int_{0}^{\infty}\left[r\left(s,-|n|^{2}\right)\right]^{2} d s=\frac{|n|^{2}}{1+|n|^{2}}
$$

and hence $C_{b}=1$. 
ii) In case $b(t)=t e^{-t}$ we obtain, after a calculation using, e.g., 6 6, Formula 2.662 $(2)]$,

$$
|n|^{2} \int_{0}^{\infty}\left[r\left(s,-|n|^{2}\right)\right]^{2} d s=\frac{|n|^{2}}{4+4|n|^{2}}
$$

and hence $C_{b}=\frac{1}{4}$.

By a solution $X(t, \theta)$ to the stochastic Volterra equation (1.1) we will understand a process $X$ taking values in the space $S^{\prime}\left(T^{d}\right)$ and satisfying (1.1). The following is our main result.

Theorem 3.3. Assume $b \in L^{1}\left(\mathbb{R}_{+}\right)$is admissible for (1.1). Then, (1.1) has an $H^{\alpha+1}\left(T^{d}\right)$-valued solution if and only if the Fourier coefficients $\left(\gamma_{n}\right)$ of the covariance $\Gamma$ satisfy

$$
\sum_{n \in \mathbb{Z}^{d}} \gamma_{n}\left(1+|n|^{2}\right)^{\alpha}<\infty
$$

In particular, (1.1) has an $L^{2}\left(T^{d}\right)$-valued solution if and only if

$$
\sum_{n \in \mathbb{Z}^{d}} \frac{\gamma_{n}}{1+|n|^{2}}<\infty
$$

Proof. We shall use the representation (2.4) for the Wiener process $W_{\Gamma}(t, \theta)$ with respect to the basis: $1, \cos (n, \theta), \sin (n, \theta)$, where $n \in \mathbb{Z}_{s}^{d}$ and $\theta \in T^{d}$. Denote by $W_{n}(t, \theta):=\cos (n, \theta) \beta_{n}^{1}(t)+\sin (n, \theta) \beta_{n}^{2}(t), n \in \mathbb{Z}_{s}^{d}$, that is, the $n$-th element in the expansion (2.4). Equation (1.1) may be solved coordinatewise as follows.

Assume that

$$
X(t, \theta)=\sum_{n \in \mathbb{Z}_{s}^{d}}\left[\cos (n, \theta) X_{n}^{1}(t)+\sin (n, \theta) X_{n}^{2}(t)\right]+X_{0}(t) .
$$

Introducing (3.4) into (1.1), we obtain

$$
\begin{aligned}
\cos (n, \theta) & X_{n}^{1}(t)+\sin (n, \theta) X_{n}^{2}(t) \\
= & -|n|^{2} \int_{-\infty}^{t} b(t-s)\left[\cos (n, \theta) X_{n}^{1}(s)+\sin (n, \theta) X_{n}^{2}(s)\right] d s \\
& +\sqrt{2 \gamma_{n}} \int_{-\infty}^{t} b(t-s)\left[\cos (n, \theta) \beta_{n}^{1}(s)+\sin (n, \theta) \beta_{n}^{2}(s)\right] d s,
\end{aligned}
$$

or, equivalently

$$
\begin{aligned}
{[\cos (n, \theta), \sin (n, \theta)]\left[\begin{array}{c}
X_{n}^{1}(t) \\
X_{n}^{2}(t)
\end{array}\right] } & =-|n|^{2} \int_{-\infty}^{t} b(t-s)[\cos (n, \theta), \sin (n, \theta)]\left[\begin{array}{c}
X_{n}^{1}(s) \\
X_{n}^{2}(s)
\end{array}\right] d s \\
& +\sqrt{2 \gamma_{n}} \int_{-\infty}^{t} b(t-s)[\cos (n, \theta), \sin (n, \theta)]\left[\begin{array}{l}
d \beta_{n}^{1}(s) \\
d \beta_{n}^{2}(s)
\end{array}\right] .
\end{aligned}
$$

Denoting

$$
X_{n}(t):=\left[\begin{array}{c}
X_{n}^{1}(t) \\
X_{n}^{2}(t)
\end{array}\right] \quad \text { and } \quad \beta_{n}(t):=\left[\begin{array}{c}
\beta_{n}^{1}(t) \\
\beta_{n}^{2}(t)
\end{array}\right]
$$

we arrive at the equation

$$
X_{n}(t)=-|n|^{2} \int_{-\infty}^{t} b(t-s) X_{n}(s) d s+\sqrt{2 \gamma_{n}} \int_{-\infty}^{t} b(t-s) d \beta_{n}(s) .
$$


Taking the Laplace transform in $t$, and making use of (3.1) with $\mu=-|n|^{2}$ and (3.2), we get the following solution to (3.5):

$$
X_{n}(t)=\int_{-\infty}^{t} r\left(t-s,-|n|^{2}\right) \sqrt{2 \gamma_{n}} d \beta_{n}(s)=\int_{0}^{\infty} r\left(s,-|n|^{2}\right) \sqrt{2 \gamma_{n}} d \beta_{n}(t-s) .
$$

Hence, we deduce the following explicit formula for the solution to (1.1):

$$
\begin{aligned}
X(t, \theta)=\sqrt{\gamma_{0}} \beta_{0}(t) & +\sum_{n \in \mathbb{Z}_{s}^{d}} \sqrt{2 \gamma_{n}}\left[\cos (n, \theta) \int_{0}^{\infty} r\left(s,-|n|^{2}\right) d \beta_{n}^{1}(t-s)\right. \\
& \left.+\sin (n, \theta) \int_{0}^{\infty} r\left(s,-|n|^{2}\right) d \beta_{n}^{2}(t-s)\right] .
\end{aligned}
$$

From definition of the space $H^{\alpha}$ and condition (2.5) the process $X(t) \in H^{\alpha+1}, P$ almost surely, if and only if

$$
\begin{aligned}
\sum_{n \in \mathbb{Z}^{d}}\left(1+|n|^{2}\right)^{\alpha+1} \gamma_{n}\left[\left(\int_{-\infty}^{t} r(t-s,\right.\right. & \left.\left.-|n|^{2}\right) d \beta_{n}^{1}(s)\right)^{2} \\
& \left.+\left(\int_{-\infty}^{t} r\left(t-s,-|n|^{2}\right) d \beta_{n}^{2}(s)\right)^{2}\right]<\infty .
\end{aligned}
$$

In the representation (2.4) of $W_{\Gamma}$, processes $\beta_{0}, \beta_{n}^{1}, \beta_{n}^{2}, n \in \mathbb{Z}_{s}^{d}$, are independent real Brownian motions. So, the stochastic integrals in (3.7) are independent Gaussian random variables, too. Hence, (3.7) holds $P$-almost surely if and only if

$$
\begin{aligned}
\sum_{n \in \mathbb{Z}^{d}}\left(1+|n|^{2}\right)^{\alpha+1} \gamma_{n} \mathbb{E}\left[\left(\int_{-\infty}^{t} r(t-\right.\right. & \left.\left.s,-|n|^{2}\right) d \beta_{n}^{1}(s)\right)^{2} \\
& \left.+\left(\int_{-\infty}^{t} r\left(t-s,-|n|^{2}\right) d \beta_{n}^{2}(s)\right)^{2}\right]<\infty,
\end{aligned}
$$

or equivalently, using $L^{2}$ isometry of stochastic integrals, if and only if

$$
\sum_{n \in \mathbb{Z}^{d}}\left(1+|n|^{2}\right)^{\alpha+1} \gamma_{n} \int_{0}^{\infty}\left[r\left(s,-|n|^{2}\right)\right]^{2} d s<\infty .
$$

Since $b$ is admissible for (1.1), we conclude that (3.9) holds if and only if

$$
\sum_{n \in \mathbb{Z}^{d}}\left(1+|n|^{2}\right)^{\alpha+1} \frac{\gamma_{n}}{|n|^{2}}<\infty
$$

and the proof is complete.

Concerning uniqueness, we have the following result.

Proposition 3.4. Assume $b \in L^{1}\left(\mathbb{R}_{+}\right)$is admissible for (1.1) and the following conditions hold:

(i) $\quad \sum_{n \in \mathbb{Z}^{d}} \gamma_{n}\left(1+|n|^{2}\right)^{\alpha}<\infty$,

(ii) $\quad\{1 / \tilde{b}(i k)\}_{k \in \mathbb{Z}} \subset \mathbb{C} \backslash\left\{-|n|^{2}: n \in \mathbb{Z}^{d}\right\}$.

Then, (1.1) has a unique $H^{\alpha+1}\left(T^{d}\right)$-valued solution.

Proof. Let $X(t, \theta)$ be a solution of

$$
X(t, \theta)=\int_{-\infty}^{t} b(t-s) \Delta X(s, \theta) d s .
$$


Taking Fourier transform in $\theta$ and denoting by $X_{n}(t)$ the $n$-th Fourier coefficient of $X(t, \theta)$ ( $t$ fixed), we obtain

$$
X_{n}(t)=-|n|^{2} \int_{-\infty}^{t} b(t-s) X_{n}(s) d s
$$

for all $n \in \mathbb{Z}^{d}$. Now taking the Laplace transform in $t$, we get that the Fourier coefficients of $X_{n}(t)$ ( $n$ fixed) satisfy

$$
\left(1+|n|^{2} \tilde{b}(i k)\right) \hat{X}_{n}(k)=0
$$

for all $k \in \mathbb{Z}$. According to (ii) we obtain that $\hat{X}_{n}(k)=0$ for all $k \in \mathbb{Z}$ and all $n \in \mathbb{Z}^{d}$. Hence, the assertion follows by uniqueness of the Fourier transform.

The following corollaries are an immediate consequence of Theorem 3.3. The arguments are the same as in [8. Here we give the proof for the sake of completeness.

Corollary 3.5. Suppose $b \in L^{1}\left(\mathbb{R}_{+}\right)$is admissible for (1.1) and assume $\Gamma \in$ $L^{2}\left(T^{d}\right)$. Then the integro-differential stochastic equation (1.1) has a solution with values in $L^{2}\left(T^{d}\right)$ for $d=1,2,3$.

Proof. We have to check (3.3) with $\alpha=-1$. Note, that if $\Gamma \in L^{2}\left(T^{d}\right)$, then $\hat{\Gamma}=\left(\gamma_{n}\right) \in l^{2}\left(\mathbb{Z}^{d}\right)$. Consequently

$$
\sum_{n \in \mathbb{Z}^{d}} \frac{\gamma_{n}}{1+|n|^{2}} \leq\left(\sum_{n \in \mathbb{Z}^{d}} \gamma_{n}^{2}\right)^{1 / 2}\left(\sum_{n \in \mathbb{Z}^{d}} \frac{1}{\left(1+|n|^{2}\right)^{2}}\right)^{1 / 2} .
$$

But $\sum_{n \in \mathbb{Z}^{d}} \gamma_{n}^{2}<\infty$ and

$$
\sum_{n \in \mathbb{Z}^{d}} \frac{1}{\left(1+|n|^{2}\right)^{q}}<\infty \text { if and only if } 2 q>d .
$$

Hence, the result follows.

Corollary 3.6. Suppose $b \in L^{1}\left(\mathbb{R}_{+}\right)$is admissible for (1.1) and assume $\hat{\Gamma} \in l^{p}\left(\mathbb{Z}^{d}\right)$ for $1<p \leq 2$. Then the integro-differential stochastic equation (1.1) has a solution with values in $L^{2}\left(T^{d}\right)$ for all $d<\frac{2 p}{p-1}$.

Proof. Note that

$$
\sum_{n \in \mathbb{Z}^{d}} \frac{\gamma_{n}}{1+|n|^{2}} \leq\left(\sum_{n \in \mathbb{Z}^{d}} \gamma_{n}{ }^{p}\right)^{1 / p}\left(\sum_{n \in \mathbb{Z}^{d}} \frac{1}{\left(1+|n|^{2}\right)^{q}}\right)^{1 / q}
$$

where $\frac{1}{p}+\frac{1}{q}=1$. Hence the result follows from (3.10) with $q=\frac{p}{p-1}$.

For $\alpha=-1$, the condition (3.3) can be written as follows.

Theorem 3.7. Let $b$ be admissible for (1.1). Assume that the covariance $\Gamma$ is not only a positive definite distribution but is also a non-negative measure. Then (1.1) has a $L^{2}\left(T^{d}\right)$-valued solution if and only if

$$
\left(\Gamma, G_{d}\right)<+\infty,
$$

where

$$
G_{d}(x)=\sum_{n \in \mathbb{Z}^{d}} \int_{0}^{+\infty} \frac{1}{\sqrt{(4 \pi t)^{d}}} e^{-t} e^{-\frac{|x+2 \pi n|^{2}}{t}} d t, \quad x \in T^{d}
$$


The proof of Theorem 3.7 is the same that for Theorem 2, part 2) in [8], so we omit it.

The functions $G_{d}(x)$ are apparently well known. If $d=1$, then $G_{1}$ is a positive continuous function. If $d=2, G_{2}$ is continuous outside 0 with singularity at 0 of the form $c \log \frac{1}{|x|}, c>0$. For $d>2, G_{d}(x) \sim c \frac{1}{|x|^{d-2}}$ as $x \rightarrow 0$. For more details concerning the function $G_{d}$ we refer to [9, Proposition 3] and [11].

From the above properties of functions $G_{d}$ defined by (3.12) and the condition (3.11) we obtain the following result (see 9, Theorem 4]).

Corollary 3.8. Assume that $\Gamma$ is a non-negative measure and $b$ is admissible. Then (1.1) has function valued solutions:

i) for all $\Gamma$ if $d=1$;

ii) for exactly those $\Gamma$ for which $\int_{|\theta| \leq 1} \ln |\theta| \Gamma(d \theta)<+\infty$ if $d=2$;

iii) for exactly those $\Gamma$ for which $\int_{|\theta| \leq 1} \frac{1}{|\theta|^{d-2}} \Gamma(d \theta)<+\infty$ if $d \geq 3$.

In what follows, we will see that formula (3.6) also implies that $X$ is Hölder continuous with respect to $t$. In order to do that, we need assumptions very similar to those in 2 .

Hypothesis $\mathbf{( H )}$. Assume that there exist $\delta \in(0,1)$ and $C_{\delta}>0$ such that, for all $s \in(-\infty, t)$ we have:

(i) $\quad \int_{s}^{t}\left[r\left(t-\tau,-|n|^{2}\right)\right]^{2} d \tau \leq C_{\delta}|n|^{2(\delta-1)}|t-s|^{\delta} ;$

(ii) $\quad \int_{-\infty}^{s}\left[r\left(t-\tau,-|n|^{2}\right)-r\left(s-\tau,-|n|^{2}\right)\right]^{2} d \tau \leq C_{\delta}|n|^{2(\delta-1)}|t-s|^{\delta}$.

Proposition 3.9. Assume that $\sum_{n \in \mathbb{Z}^{d}} \frac{\gamma_{n}}{1+|n|^{2}}<+\infty$. Under Hypothesis $(\mathrm{H})$, the trajectories of the solution $X$ to (1.1) are almost surely $\eta$-Hölder continuous with respect to $t$, for every $\eta \in(0, \delta / 2)$.

Proof. From the expansion (3.6) and properties of stochastic integral, we have

$$
\begin{aligned}
& \mathbb{E}\|X(t, \theta)-X(s, \theta)\|_{L^{2}}^{2}=\mathbb{E} \| \sqrt{\gamma_{0}}\left(\beta_{0}(t)-\beta_{0}(s)\right) \\
& \quad+\sum_{n \in \mathbb{Z}_{s}^{d}} \sqrt{2 \gamma_{n}}\left[\cos (n, \theta)\left(\int_{-\infty}^{t} r\left(t-\tau,-|n|^{2}\right) d \beta_{n}^{1}(\tau)-\int_{-\infty}^{s} r\left(s-\tau,-|n|^{2}\right) d \beta_{n}^{1}(\tau)\right)\right. \\
& \left.\quad \quad+\sin (n, \theta)\left(\int_{-\infty}^{t} r\left(t-\tau,-|n|^{2}\right) d \beta_{n}^{2}(\tau)-\int_{-\infty}^{s} r\left(s-\tau,-|n|^{2}\right) d \beta_{n}^{2}(\tau)\right)\right]||_{L^{2}}^{2} \\
& =(2 \pi)^{d}\left(\gamma_{0}|t-s|\right. \\
& \left.\quad+\sum_{n \in \mathbb{Z}_{s}^{d}} 2 \gamma_{n}\left[\int_{-\infty}^{s}\left[r\left(t-\tau,-|n|^{2}\right)-r\left(s-\tau,-|n|^{2}\right)\right]^{2} d \tau+\int_{s}^{t} r^{2}\left(t-\tau,-|n|^{2}\right) d \tau\right]\right) .
\end{aligned}
$$

According to assumptions (i) and (ii) of the Hypothesis $(\mathrm{H})$, we have

$$
\mathbb{E}|| X(t, \theta)-X(s, \theta) \|_{L^{2}}^{2} \leq C_{\delta} \sum_{n \in \mathbb{Z}_{s}^{d}} 2 \gamma_{n}|n|^{2(\delta-1)}|t-s|^{\delta} .
$$

Because $X$ is a Gaussian process, then for any $m \in N$, there exists a constant $C_{m}>0$ that

$$
\mathbb{E}|| X(t, \theta)-X(s, \theta) \|_{L^{2}}^{2 m} \leq C_{m}\left[C_{\delta} \sum_{n \in \mathbb{Z}_{s}^{d}} 2 \gamma_{n}|n|^{2(\delta-1)}\right]^{m}|t-s|^{m \delta} .
$$


Taking $m$ such that $m \delta>1$ and using Kolmogorov's criterion for continuity (see, e.g., [18]), we obtain that the solution $X(t, \theta)$ is $\eta$-Hölder continuous, with respect to $t$, for $\eta=\delta / 2-1 /(2 m)$.

Example 3.10. Let us consider the particular case $b(t)=e^{-t}, t \geq 0$. Then, by previous considerations, $r\left(t,-|n|^{2}\right)=e^{\left(-1-|n|^{2}\right) t}$. One can check that in this case Hypothesis (H) is fulfilled.

Remark 3.11. We observe that condition (i) in Hypothesis (H) is the same as

$$
|n|^{2} \int_{0}^{t}\left[r\left(s,-|n|^{2}\right)\right]^{2} d s \leq C_{\delta}|n|^{2 \delta}|t|^{\delta},
$$

and hence it is nearly equivalent to saying that the function $b$ is admissible.

\section{ACKNOWLEDGEMENT}

The authors would like to thank the anonymous referee for the careful reading of the manuscript. The valuable remarks made numerous improvements throughout.

\section{REFERENCES}

[1] R. Adams, Sobolev Spaces, Academic Press, New York, 1975. MR0450957 (56:9247)

[2] Ph. Clément, G. Da Prato, Some results on stochastic convolutions arising in Volterra equations perturbed by noise, Rend. Math. Acc. Lincei. s. 9, 7, (1996) 147-153. MR 1454409 (98e:60106)

[3] Ph. Clément, G. Da Prato, Existence and regularity results for an integral equation with infinite delay in a Banach space, Integral Equations and Operator Theory, 11, (1988) 480500. MR0950513 (89f:45019)

[4] R. Dalang, N. Frangos, The stochastic wave equation in two spatial dimensions, The Annals of Probability 26, (1998) 187-212. MR.1617046 (99c:60127)

[5] C. Gasquet, P. Witomski, Fourier Analysis and Applications, Springer-Verlag, New York, Berlin, 1999. MR1657104 (99h:42003)

[6] I.S. Gradshteyn, I.M. Ryzhik, Table of Integrals, Series and Products, Corrected and Enlarged Revision, Academic Press, San Diego, New York, London, Tokyo, 1980.

[7] G. Gripenberg, S.-O. Londen, O. Staffans, Volterra Integral and Functional Equations, Encyclopedia of Mathematics and its Applications 34, Cambridge University Press, CambridgeNew York, 1990. MR1050319 (91c:45003)

[8] A. Karczewska, J. Zabczyk, A note on stochastic wave equations, G. Lumer and L. Weis (eds.), Evolution equations and their applications in physical and life sciences, Marcel Dekker, Lect. Notes Pure Appl. Math. 215, 501-511 (2001). MR1818028(2002b:60112)

[9] A. Karczewska, J. Zabczyk, Stochastic PDE's with function-valued solutions. Ph. Clément (ed.) et al., Infinite dimensional stochastic analysis. Royal Netherlands Academy of Arts and Sciences. Verh. Afd. Natuurkd., 1. Reeks, K. Ned. Akad. Wet. 52, 197-216 (2000). MR1832378 (2002h:60132)

[10] A. Karczewska, J. Zabczyk, Regularity of solutions to stochastic Volterra equations, Rend. Math. Acc. Lincei. s. 9, 11, (2001) 141-154. MR1841688 (2002f:60124)

[11] N.S. Landkof, Foundations of modern potential theory, Springer-Verlag, Berlin, 1975. MR0350027 (50:2520)

[12] A. Millet, M. Sanz-Solé, A stochastic wave equation in two space dimension: smoothness of the law, The Annals of Probability 27 (2), (1999) 803-844. MR1698971 (2001e:60130)

[13] C. Mueller, Long time existence for the wave equation with a noise term, The Annals of Probability 25 (1), (1997) 133-151. MR1428503 (98b:60113)

[14] J.W. Nunziato, On heat conduction in materials with memory, Quart. Appl. Math. 29, (1971) 187-204. MR0295683 (45:4749)

[15] J. Prüss, Evolutionary Integral Equations and Applications. Monographs Math., vol. 87, Birkhäuser Verlag, Basel, 1993. MR.1238939(94h:45010) 
[16] M. Reed, B. Simon, Methods of modern mathematical physics, Vol. II, Academic Press, New York, 1975.

[17] L. Schwartz, Méthodes mathématiques pour les sciences physiques, Hermann, Paris, 1965. MR0143360 (26:919)

[18] D. Stroock, Probability Theory, an analytic view, Cambridge University Press, Cambridge, 1993. MR1267569 (95f:60003)

Department of Mathematics, University of Zielona Góra, ul. Szafrana 4a, 65-246 Zielona Góra, Poland

E-mail address: A.Karczewska@im.uz.zgora.pl

Departamento de Matemática, Facultad de Ciencias, Universidad de Santiago de Chile, Casilla 307-Correo 2, Santiago, Chile

E-mail address: clizama@lauca.usach.cl 